\title{
Magnetic response of magnetic molecules with non-collinear local $d$-tensors
}

\author{
J. Schnack ${ }^{1 *}$ \\ ${ }^{1}$ Universität Bielefeld, Fakultät für Physik, Postfach 100131, D-33501 Bielefeld, Germany
}

Received May 26, 2009, in final form June 15, 2009

\begin{abstract}
Investigations of molecular magnets are driven both by prospective applications in future storage technology or quantum computing as well as by fundamental questions. Nowadays numerical simulation techniques and computer capabilities make it possible to investigate spin Hamiltonians with realistic arrangements of local anisotropy tensors. In this contribution I will discuss the magnetic response of a small spin system with special emphasis on non-collinear alignments of the local anisotropy axes.
\end{abstract}

Key words: magnetic molecules, single-ion anisotropy, exact diagonalization

PACS: $75.50 . X x, 75.10 . J m, 75.40 . C x, 75.40 . M g$

\section{Introduction}

Since the early investigations of $\mathrm{Mn}_{12}$-acetate [1], single molecule magnets (SMM) have been at the heart of the investigations of magnetic molecules worldwide. This is due to their properties which are governed by the anisotropy barrier, as there is observed slow relaxation of the magnetization as well as spin tunneling through the barrier [2-5]. From the chemical point of view, it is striking that until recently no compound could be synthesized with a higher anisotropy barrier which moved hopes of easy application into the more distant future. Theoretical estimates pointed out that this might be due to very general reasons [6,7]. A recently synthesized manganese compound put an end to more than 20 years of search [8].

On the theory side, numerically exact evaluations of spin Hamiltonians including anisotropic terms turned out to be limited to rather small systems such as for instance an antiferromagnetically coupled $\mathrm{Ni}_{4}$ compound [9-12], which was investigated in great detail or another but ferromagnetically coupled $\mathrm{Ni}_{4}$ [13]. With great numerical effort the exchange constants of $\mathrm{Mn}_{12}$-acetate could be determined [14], but usually one resorts to models where the interacting spin system is replaced by one large spin in its effective ligand field (giant spin approximation). But thanks to the technical progress detailed numerical studies of larger molecules are affordable nowadays. First examples are given by the simulation of the new $\mathrm{Mn}_{6}$ compound with record anisotropy barrier [15] and by the investigation of a $\mathrm{Mn}_{6} \mathrm{Fe}$ compound [16]. Whereas [15] restricts its parameter space to collinear local anisotropy axes, [16] considers non-collinear ones.

In this contribution the recently developed procedures will be used to discuss the effect of noncollinear anisotropy axes on the magnetic response of a fictitious molecular compound. In order not to make the situation too complicated I will restrict the discussion to a triangular arrangement of equal spins of $s=1$ with a molecular $C_{3}$ symmetry. Then the magnetization will be discussed for three typical scenarios given by the possible ratios of exchange and anisotropy: $|J|>|d|,|J|=|d|$, and $|J|<|d|$.

*jschnack@physik.uni-bielefeld.de 


\section{Hamiltonian and evaluation}

\subsection{Hamiltonian}

It turns out that many magnetic molecules can be well described by a Heisenberg model which takes the super exchange between the moments of the (typically $3 d$ ) ions into account. In this strong coupling limit anisotropic terms appear as perturbations and are thus often taken care of by perturbation theory. But for single molecule magnets, that might e. g. contain Mn(III) or V(III) ions, the situation might be reversed or anisotropy and exchange are at least of the same order. Therefore, I would like to treat both on the same footing right from the beginning. In the following Hamiltonian,

$$
\underset{\sim}{H}(\vec{B})=-\sum_{i, j} J_{i j}{\underset{\sim}{\vec{s}}}_{i} \cdot{\underset{\sim}{\vec{s}}}_{j}+\sum_{i} d_{i}\left(\vec{e}_{i} \cdot{\underset{\sim}{\vec{s}}}_{i}\right)^{2}+\mu_{B} \vec{B} \cdot \sum_{i} \mathbf{g}_{i} \cdot \vec{\sim}_{i},
$$

the first term models the isotropic Heisenberg exchange interaction. A negative $J_{i j}$ corresponds to an antiferromagnetic coupling of spins at sites $i$ and $j$. The second term models the local anisotropy tensors by their major principal axis which points along $\vec{e}_{i}$. Depending on the sign of $d_{i}$ this is an easy $\left(d_{i}<0\right)$ or hard $\left(d_{i}>0\right)$ axis. The neglected two other principal axes of the local $\mathbf{d}$-tensor $(e-$ terms) are usually much smaller. The last term provides the interaction with the applied magnetic field. Here $\mathbf{g}_{i}$ is the local $g$-tensor, which might also be anisotropic.

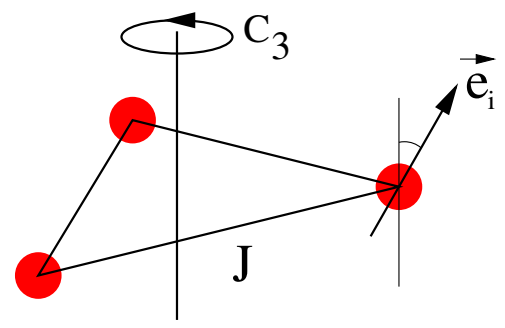

Figure 1. Configuration of the fictitious $C_{3}$-symmetric molecule discussed in this article. The remaining parameters of the model are the exchange coupling $J$, the strength of the local anisotropy $d$, and the angle $\vartheta$ of the local anisotropy axes with respect to the $C_{3}$ axis.

Since in this contribution the effect of the non-collinearity of the local anisotropy axes shall be discussed, the Hamiltonian will be restricted to a simplified spin system, that is depicted in figure 1. Three spins $s=1$ of a fictitious $C_{3}$-symmetric molecule will be considered that interact with one and the same exchange $J$. The local anisotropy axes are also related by the $C_{3}$ symmetry, so that the anisotropy can be modeled by one strength $d$ and one azimuthal angle $\vartheta$. The $g$-tensors are assumed to be isotropic and of value 2 .

\subsection{Evaluation}

Since the various parts of Hamiltonian (1) do not commute in general all eigenvalues and eigenvectors have to be calculated for each magnetic field $\vec{B}$, i. e. for each strength and direction of the field. The magnetization is then evaluated using all eigenvectors. I would like to mention that it is also possible to obtain the magnetization without using eigenvectors by numerical differentiation of the energy eigenvalues $E_{\nu}(\vec{B})$, which are functions of $\vec{B}$.

Very often the investigated substance is only available as a powder. Then an orientational average has to be performed in order to be able to compare to experiments. Since this average cannot be performed analytically, one sums over a finite set of special directions. In our evaluations we use for each absolute value $B=|\vec{B}|$ special sets of directions given by points on the unit sphere. These sets are called Lebedev-Laikov grids [17]. The directions, which contribute with various weights, and their total number are chosen such that the angular integration of polynomials $x^{k} \times y^{l} \times z^{m}$, where $k+l+m \leqslant 131$, can be performed with a relative accuracy of $2^{-14}$ [17]. In our 
evaluations we normally use a Lebedev-Laikov grid with 50 points. These points can be generated with publicly available software [18]. Equivalently one may cover the unit sphere with the vertices of regular bodies such as an octahedron, a cube, or a dodecahedron. For practically all examples we evaluated in the past we can say that averaging over the directions given by the vertices of a dodecahedron is as accurate as averaging over a Lebedev-Laikov grid with 50 points. On the contrary, averaging only over a smaller number of directions like $\pm x, \pm y$, and $\pm z$ is insufficient.

\section{Anisotropy versus coupling}

In the following main part the magnetic response of the spin system shown in figure 1 will be discussed for three scenarios: $|J|>|d|,|J|=|d|$, and $|J|<|d|$. In most cases the figures show powder averaged magnetizations for $\vartheta=0,10,20,30,40,50,60,70,80,90$ degrees. Solid curves correspond to $\vartheta=0,10,20,30$ degrees, dashed ones to $\vartheta=40,50,60,70$ degrees, and dashed-dotted curves depict $\vartheta=80,90$ degrees. In order to pronounce the details, all magnetization curves are shown at a low temperature of $T=0.1|J|$.

\subsection{Strong coupling limit}
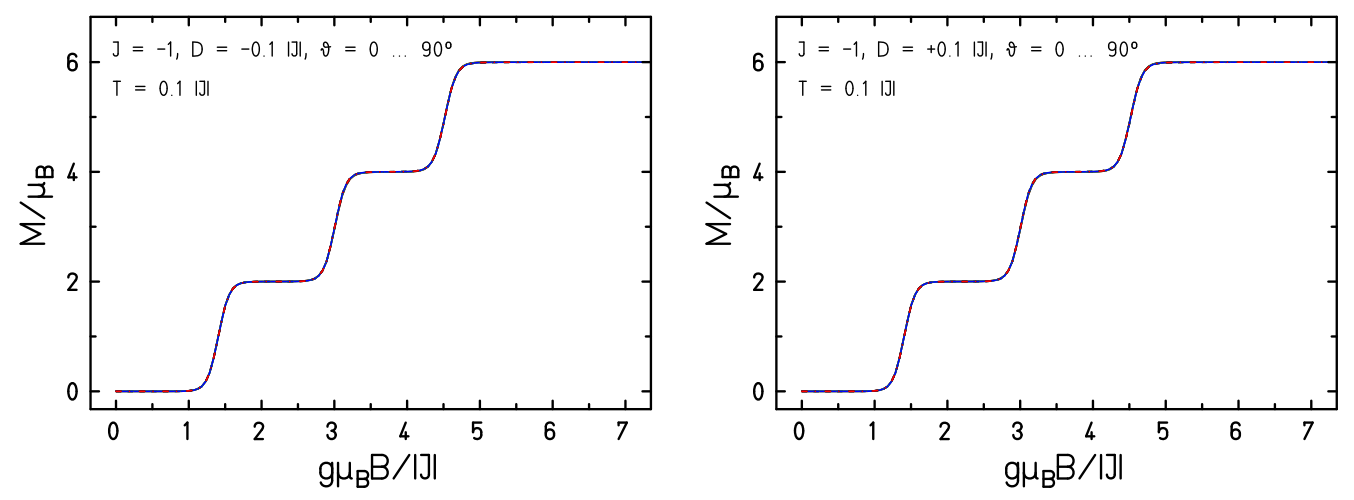

Figure 2. Magnetization for antiferromagnetic coupling $J=-1$ and easy axis anisotropy $d=$ $-0.1|J|$ (l.h.s.) as well as hard axis anisotropy $d=+0.1|J|$ (r.h.s.). $\vartheta=0,10,20,30,40,50,60$, $70,80,90$ degrees.
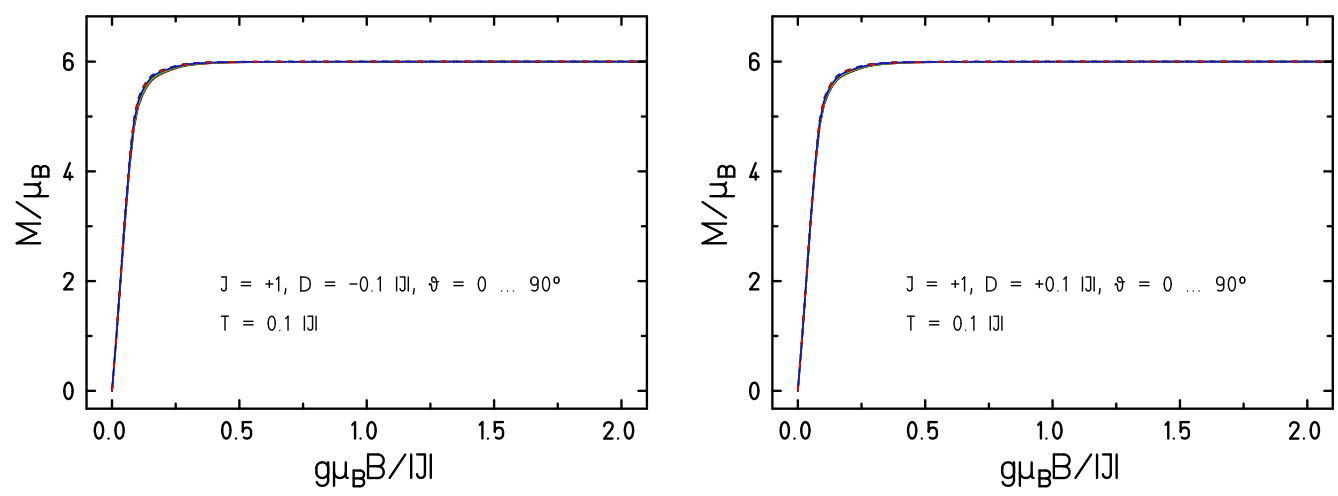

Figure 3. Magnetization for ferromagnetic coupling $J=+1$ and easy axis anisotropy $d=-0.1|J|$ (l.h.s.) as well as hard axis anisotropy $d=+0.1|J|$ (r.h.s.). $\vartheta=0,10,20,30,40,50,60,70,80,90$ degrees.

In the strong coupling limit the exchange energy is much bigger than the anisotropy energy, i. e. $|J|>|d|$. This is the case for many molecules, e. g. antiferromagnetically coupled iron or 
chromium rings [19-28]. What these systems have in common is that a description in terms of a plain Heisenberg Hamiltonian often provides a very good approximation [29-32]. If needed the anisotropic terms can be included via perturbation theory.

In figures 2 and 3 the magnetization curves are shown for combinations of anti-/ferromagnetic coupling $J=\mp 1$ and easy/hard axis anisotropy $d=\mp 0.1|J|$. In the antiferromagnetic case (figure 2) the low-temperature magnetization curve is a staircase due to the successive ground-state level crossings in the growing magnetic field whereas in the ferromagnetic case the magnetization curve is practically given by the Brillouin function of the ground state $\operatorname{spin} S=3$. Since the anisotropy is small in magnitude its angular variation is not noticeable.

\subsection{Intermediate coupling}
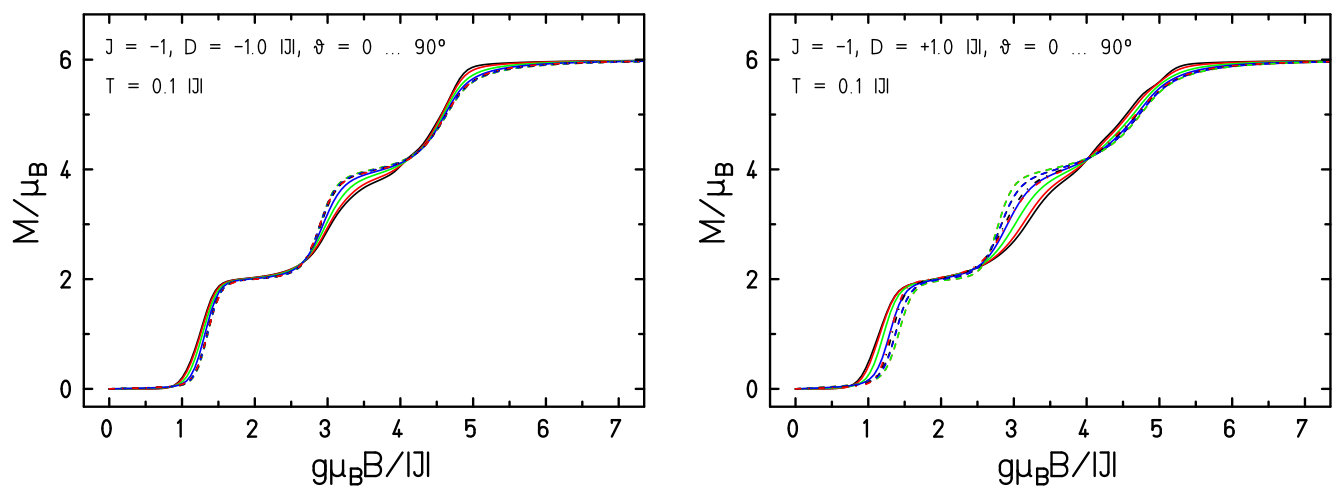

Figure 4. Magnetization for antiferromagnetic coupling $J=-1$ and easy axis anisotropy $d=$ $-|J|$ (l.h.s.) as well as hard axis anisotropy $d=|J|$ (r.h.s.). $\vartheta=0,10,20,30,40,50,60,70,80$, 90 degrees.
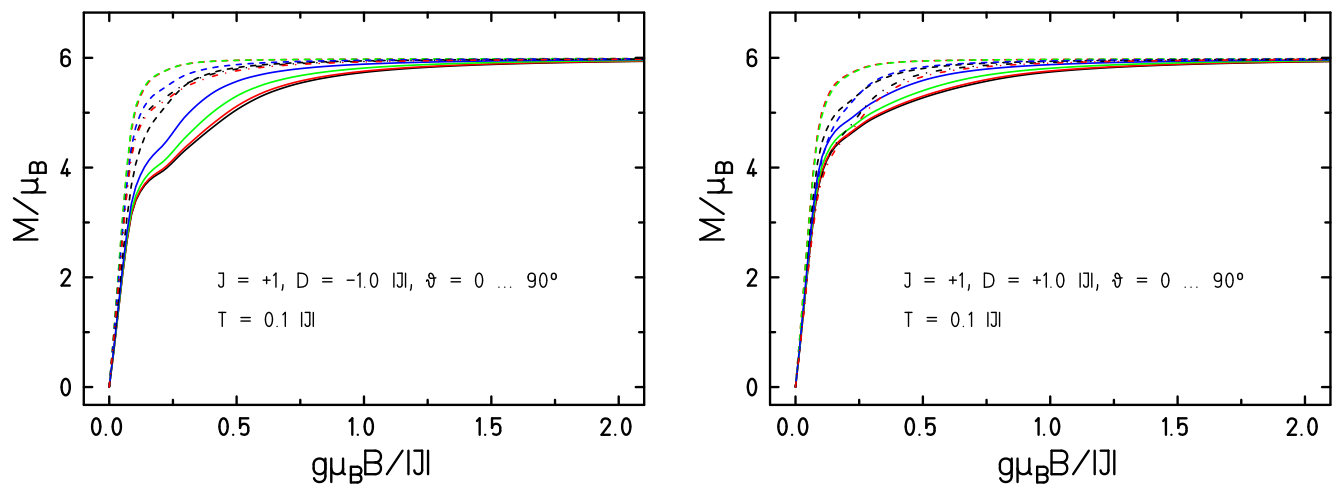

Figure 5. Magnetization for ferromagnetic coupling $J=+1$ and easy axis anisotropy $d=-|J|$ (l.h.s.) as well as hard axis anisotropy $d=|J|$ (r.h.s.). $\vartheta=0,10,20,30,40,50,60,70,80,90$ degrees.

In the intermediate coupling regime exchange and anisotropy are of the same order. In the examples presented in figure 4 and 5 they have the same amplitude $|J|=|d|$. Looking at the figures one easily notices that the now larger anisotropy smears out the angle averaged magnetization curves. Nevertheless, the underlying structure - steps for the antiferromagnetic case and Brillouin function like for the ferromagnetic case - is still clearly visible. Interestingly, the structure of the magnetization curve is most similar to the strong coupling limit for intermediate angles $\vartheta$ around $50^{\circ} \ldots 60^{\circ}$.

In detail the curves follow the following trend with varying $\vartheta$. At small $\vartheta$ (solid curves), i. e. close to the collinear configuration, the effect of the anisotropy is strong since it acts cooperatively. 
Then for intermediate $\vartheta$ (dashed curves) the effect of the anisotropy is the weakest compared to the strong coupling limit. This is understandable if one recalls that $\vartheta=\arccos (1 / \sqrt{3})=54,73^{\circ}$ is the angle of a perfect octahedral alignment of the anisotropy axes, i. e. the three axis have a pairwise angle of $90^{\circ}$. In this configuration the effect of the anisotropic terms on multiplets of the Heisenberg Hamiltonian is either canceled or rather small. This explains why both at $50^{\circ}$ and $60^{\circ}$ anisotropy effects are hardly visible. For $\vartheta>60^{\circ}$ (dashed-dotted curves) the effect of the anisotropy rises again.

\subsection{Weak coupling limit}
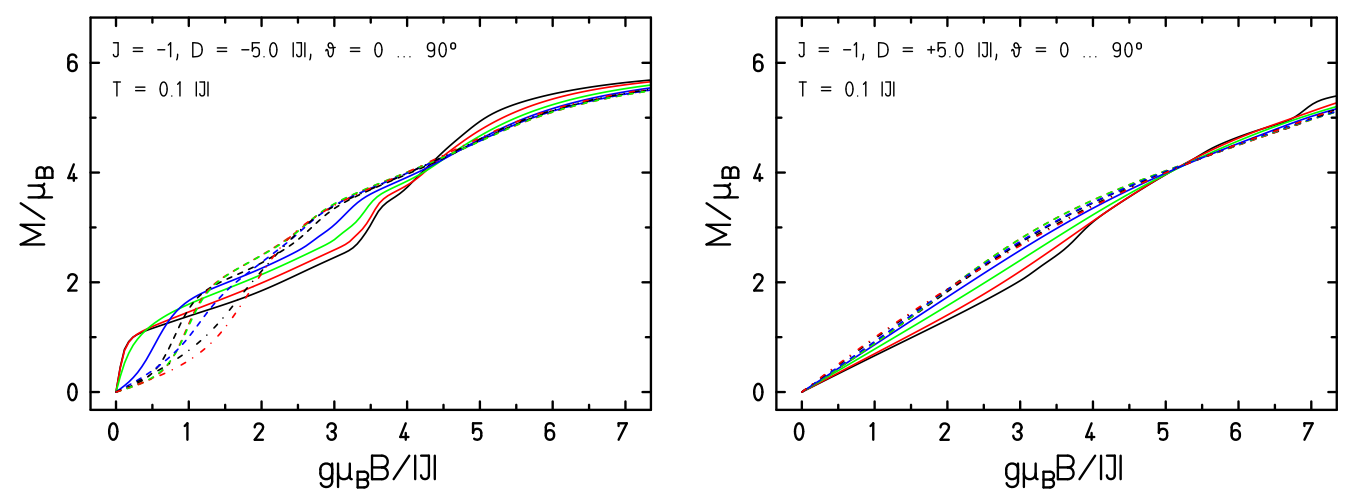

Figure 6. Magnetization for antiferromagnetic coupling $J=-1$ and easy axis anisotropy $d=$ $-5|J|$ (l.h.s.) as well as hard axis anisotropy $d=+5|J|$ (r.h.s.). $\vartheta=0,10,20,30,40,50,60,70$, 80, 90 degrees.
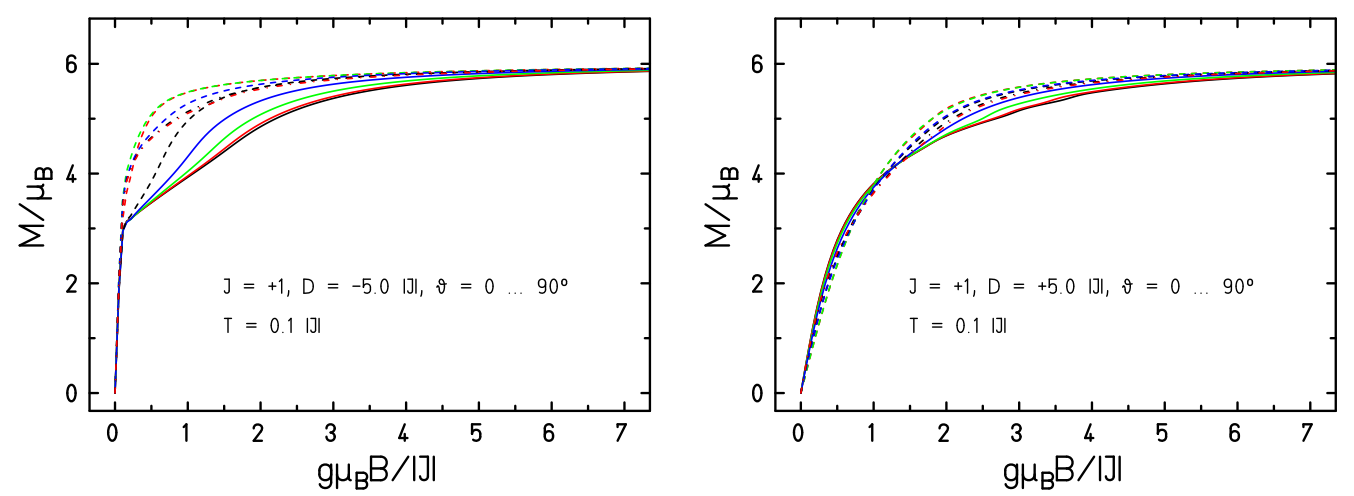

Figure 7. Magnetization for ferromagnetic coupling $J=+1$ and easy axis anisotropy $d=-5|J|$ (l.h.s.) as well as hard axis anisotropy $d=+5|J|$ (r.h.s.). $\vartheta=0,10,20,30,40,50,60,70,80,90$ degrees.

The weak coupling limit, for which the exchange energy is (much) smaller than the anisotropy energy, i. e. $|J|<|d|$, is interesting since this case seems to be relevant for an improvement of single molecule magnets (SMM). The curves in figures 6 and 7 show that the effect of the anisotropy is as expected - strong, and in the case of antiferromagnetic coupling (figure 6) very strong. Here one can say that a hard axis anisotropy together with antiferromagnetic coupling (r.h.s. of figure 6) completely destroys any structure of the magnetization curve. A (meta-) stable magnetic ground state that is separated by an anisotropy barrier from its counterpart of opposite magnetization is either not created or the low-lying spectrum is so dense that the mechanism of a barrier breaks down. Zero-field split multiplets do not exist any more and are thus an inadequate picture for such a situation. In the antiferromagnetic case with easy axis (l.h.s. of figure 6) one can at least for 
almost all collinear alignments of the anisotropy axes obtain a non-vanishing ground state moment that is stabilized by anisotropy.
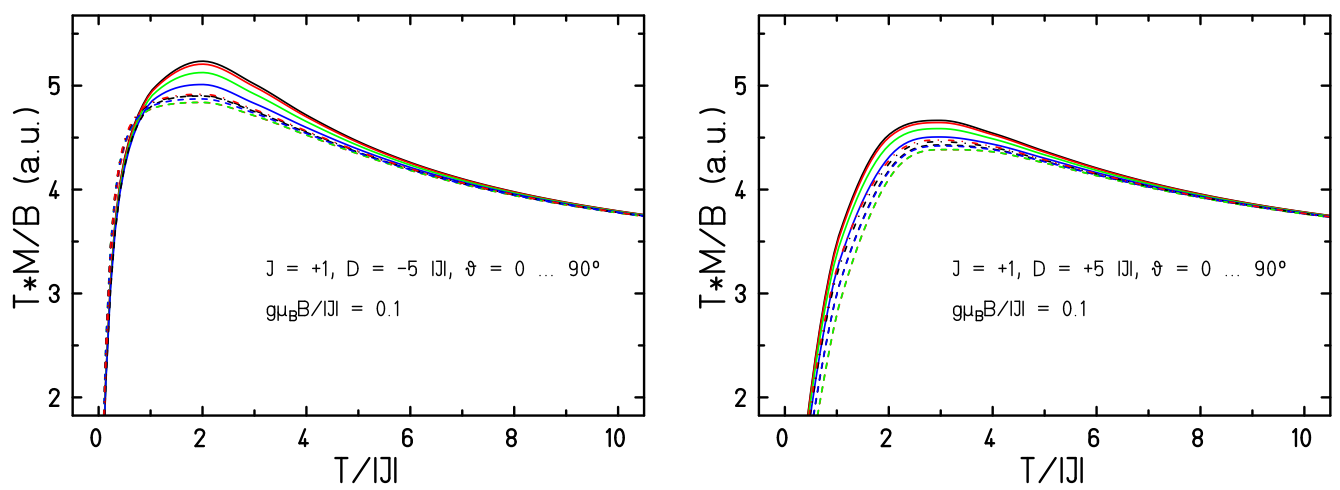

Figure 8. Magnetization as a function of temperature for $g \mu B /|J|=0.1$ for ferromagnetic coupling $J=+1$ and easy axis anisotropy $d=-5|J|$ (l.h.s.) as well as hard axis anisotropy $d=+5|J|$ (r.h.s.). $\vartheta=0,10,20,30,40,50,60,70,80,90$ degrees.

In the ferromagnetic case a hard-axis anisotropy (r.h.s. of figure 7) also has the effect to weaken the magnetization compared to a Brillouin function. The interesting case is here again the case of easy-axis anisotropy (l.h.s. of figure 7). If the magnetization for a small field, here $g \mu B /|J|=0.1$, is plotted against temperature (figure 8) one again sees that a hard-axis anisotropy tends to smear out and weaken the magnetization (r.h.s. of figure 8) whereas the curves appear sharper for easyaxis anisotropy (l.h.s. of figure 8). It is worthwhile to investigate the energy spectrum of the latter configuration in some detail and compare it to the respective cases of intermediate and strong coupling.

\subsection{Possible SMM behavior}
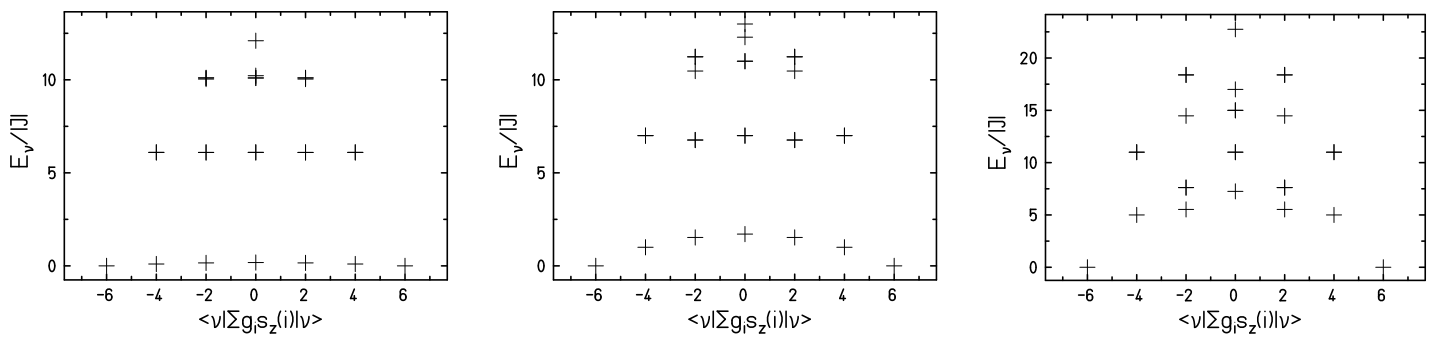

Figure 9. Energy eigenvalues at $B=0$ for $J=+1$ and $d=-0.1|J|$ (l.h.s.), $d=-|J|$ (middle), and $d=-5|J|$ (r.h.s.). $\vartheta=0$.

Regarding the question which of the cases is preferential for a good SMM, the energy eigenvalues have to be studied. This is done for the zero-field case and $J=+1$ and $d=-0.1|J|$ (l.h.s. of figure 9), $d=-|J|$ (middle of figure 9), and $d=-5|J|$ (r.h.s. of figure 9). The anisotropy axes are aligned in a collinear fashion, i. e. $\vartheta=0$, in order to act constructively. For a good SMM it would be necessary to obtain a low-lying zero-field split multiplet with both high total spin as well as high anisotropy barrier. In addition, these levels should be largely separated from higher lying levels. Looking at figure 9 one notices that in the strong coupling limit the multiplets can be well separated, but the ground-state multiplet is practically not split. In the opposite case of large easy-axis anisotropy the ground state multiplet is nicely split but energetically overlaps with other levels (but not too strong). Such a scenario was for instance discussed for the aforementioned $\mathrm{Mn}_{6}$ cluster [15]. It turns out that the intermediate case, where exchange and easy-axis anisotropy 
are of similar magnitude provides the best chances for obtaining a good SMM: the ground-state multiplet is correctly zero-field split and the higher lying states are well separated.

\section{Summary}

In this article the effect of non-collinear anisotropy axes on the magnetic response of a small spin cluster was discussed. The main result is that for good single molecule magnets, ferromagnetic coupling together with an anisotropy of collinear easy axes that is of similar magnitude is preferential. Various other scenarios have been discussed.

\section{Acknowledgement}

This work was supported by the German Science Foundation (DFG) through the research group 945 .

\section{References}

1. Lis T., Acta Chrytallogr. B, 1980, 36, 2042.

2. Thomas L., Barbara B., J. Low Temp. Phys., 1998, 113, 1055.

3. Chiorescu I., Giraud R., Jansen A.G.M., Caneschi A., Barbara B., Phys. Rev. Lett., 2000, 85, 4807.

4. Leuenberger M.N., Loss D., Phys. Rev. B 61 (2000), 21286

5. Pohjola T., Schoeller H., Phys. Rev. B, 2000, 62, 2215026

6. Waldmann O., Inorg. Chem., 2007, 46, 10035.

7. Ruiz E., Cirera J., Cano J., Alvarez S., Loose C., Kortus J., Chem. Commun., 2008, 52.

8. Milios C.J., Vinslava A., Wernsdorfer W., Moggach S., Parsons S., Perlepes S.P., Christou G., Brechin E.K., J. Am. Chem. Soc., 2007, 129, 2754.

9. Postnikov A.V., Brüger M., Schnack J., Phase Transitions, 2005, 78, 47.

10. Schnack J., Brüger M., Luban M., Kögerler P., Morosan E., Fuchs R., Modler R., Nojiri H., Rai R.C., Cao J., Musfeldt J.L., Wei X., Phys. Rev. B, 2006, 73, 094401.

11. Kortz U., Müller A., van Slageren J., Schnack J., Dalal N.S., Dressel M., Coord. Chem. Rev., 2009, 253, 2315.

12. Nehrkorn J., Höck M., Brüger M., Mutka H., Schnack J., Waldmann O., 2009 (in preparation).

13. Kirchner N., van Slageren J., Tsukerblat B., Waldmann O., Dressel M., Phys. Rev. B, 2008, 78, 094426.

14. Chaboussant G., Sieber A., Ochsenbein S., Güdel H.-U., Murrie M., Honecker A., Fukushima N., Normand B., Phys. Rev. B, 2004, 70, 104422.

15. Carretta S., Guidi T., Santini P., Amoretti G., Pieper O., Lake B., van Slageren J., Hallak F.E., Wernsdorfer W., Mutka H., Russina M., Milios C.J., Brechin E.K., Phys. Rev. Lett., 2008, 100, 157203.

16. Glaser T., Heidemeier M., Krickemeyer E., Bögge H., Stammler A., Fröhlich R., Bill E., Schnack J., Inorg. Chem., 2009, 48, 607.

17. Lebedev V.I., Laikov D.N., Dokl. Akad. Nauk, 1999, 366, 741.

18. Explanations and software to generate Lebedev-Laikov grids can be found at http://www.ccl.net/cca/software/SOURCES/FORTRAN/Lebedev-Laikov-Grids/index.shtml

19. Taft K.L., Delfs C.D., Papaefthymiou G.C., Foner S., Gatteschi D., Lippard S.J., J. Am. Chem. Soc., 1994, 116, 823 .

20. Pilawa B., Desquiotz R., Kelemen M., Weickenmeier M., Geisselman A., J. Magn. Magn. Mater., 1997, 177, 748 .

21. Julien M.-H., Jang Z., Lascialfari A., Borsa F., Horvatić M., Caneschi A., Gatteschi D., Phys. Rev. Lett., 1999, 83, 227.

22. van Slageren J., Sessoli R., Gatteschi D., Smith A.A., Helliwell M., Winpenny R.E.P., Cornia A., Barra A.L., Jansen A.G.M., Rentschler E., Timco G.A., Chem. Eur. J., 2002, 8, 277.

23. Waldmann O., Europhys. Lett., 2002, 60, 302.

24. Carretta S., van Slageren J., Guidi T., Liviotti E., Mondelli C., Rovai D., Cornia A., Dearden A.L., Carsughi F., Affronte M., Frost C.D., Winpenny R.E.P., Gatteschi D., Amoretti G., Caciuffo R., Phys. Rev. B, 2003, 67, 094405. 
25. Affronte M., Guidi T., Caciuffo R., Carretta S., Amoretti G., Hinderer J., Sheikin I., Jansen A.G.M., Smith A.A., Winpenny R.E.P., van Slageren J., Gatteschi D., Phys. Rev. B, 2003, 68, 104403.

26. Engelhardt L., Luban M., Phys. Rev. B, 2006, 73, 054430.

27. Schnelzer L., Waldmann O., Horvatić M., Ochsenbein S.T., Krämer S., Berthier C., Güdel H.U., Pilawa B., Phys. Rev. Lett., 2007, 99, 087201.

28. Furukawa Y., Kiuchi K., Kumagai K., Ajiro Y., Narumi Y., Iwaki M., Kindo K., Bianchi A., Carretta S., Santini P., Borsa F., Timco G.A., Winpenny R.E.P., Phys. Rev. B, 2009, 79, 134416.

29. Bärwinkel K., Schmidt H.-J., Schnack J., J. Magn. Magn. Mater., 2000, 212, 240.

30. Bärwinkel K., Schmidt H.-J., Schnack J., J. Magn. Magn. Mater., 2000, 220, 227.

31. Schnack J., Phys. Rev. B, 2000, 62, 14855.

32. Bärwinkel K., Hage P., Schmidt H.-J., Schnack J., Phys. Rev. B, 2003, 68, 054422.

\title{
Магнітний відгук магнітних молекул з неколінеарними локальними $d$-тензорами
}

\author{
Ю. Шнак
}

Університет Білєфельда, факультет фізики, поштова скринька 100131, D-33501 Білєфельд, Німеччина

Отримано 26 травня 2009 р., в остаточному вигляді - 15 червня 2009 р.

\begin{abstract}
Актуальність молекулярних магнетиків обумовлена як перспективами використання у майбутніх технологіях зберігання чи квантовому комп'ютингу, так і з фундаментальної точки зору. Сучасні методи чисельного моделювання та можливості комп'ютерної техніки дають змогу дослідити спінові гамільтоніани з реалістичним розташуванням тензорів локальної анізотропії. У цій статті обговорюється магнітний відгук малої спінової системи з спеціальним наголосом на неколінеарне вилаштування осей локальної анізотропії.
\end{abstract}

Ключові слова: магнітні молекули, однойонна анізотропія, точна діагоналізація

PACS: $75.50 . X x, 75.10 . J m, 75.40 . C x, 75.40 . \mathrm{Mg}$ 